\title{
A treatment approach for couples with disrupted sperm DNA integrity and recurrent ART failure
}

\author{
Alessandra Parrella ${ }^{1} \cdot$ Derek Keating $^{1} \cdot$ Stephanie Cheung ${ }^{1} \cdot$ Philip Xie $^{1} \cdot$ Joshua D. Stewart $^{1} \cdot$ Zev Rosenwaks $^{1} \cdot$ \\ Gianpiero D. Palermo ${ }^{1}$
}

Received: 3 May 2019 / Accepted: 23 July 2019 / Published online: 16 August 2019

(C) The Author(s) 2019

\begin{abstract}
Objective To test a novel method to select spermatozoa with high chromatin integrity.

Design Specimens with high sperm chromatin fragmentation (SCF) were selected by density gradient selection (DGS) and microfluidic sperm sorting (MSS).

Setting Academic medical center.

Patient(s) Ejaculates from consenting men were processed by DGS/MSS. Couples underwent ICSI cycles with spermatozoa processed by DGS/MSS. Clinical outcomes were evaluated after embryo transfer.

Intervention(s) SCF was measured by TUNEL. ICSI with spermatozoa selected by DGS and MSS was performed.

Main outcome measure(s) Fertilization, embryo implantation, and pregnancy outcomes were compared between DGS and MSS. Result(s) A total of 23 men had an average SCF of $20.7 \pm 10 \%$. After DGS and MSS, the SCF was $12.5 \pm 5 \%$ and $1.8 \pm 1 \%$, respectively. In couples who underwent ICSI, the average SCF was $28.8 \pm 9 \%$, which fell to $21.0 \pm 9 \%$ after DGS and $1.3 \pm 0.7 \%$ after MSS. Four couples underwent 11 ICSI cycles with DGS and achieved one (25\%) pregnancy that resulted in pregnancy loss. In four subsequent ICSI cycles with MSS, an ongoing clinical pregnancy rate of 50\% was achieved. Five additional couples underwent 12 cycles of ICSI with DGS. After preimplantation genetic testing for aneuploidy, $30.3 \%$ of the embryos were euploid. One pregnancy was achieved, resulting in pregnancy loss. With MSS, 31.5\% of the embryos were euploid and 4 couples obtained a pregnancy. Finally, sixteen couples underwent 20 ICSI cycles solely with MSS at our center. Of these couples, 8 had failed 13 ICSI cycles with DGS elsewhere. These couples achieved an overall implantation of 34.5\% (10/29) and a pregnancy rate of $58.8 \%(10 / 17)$.
\end{abstract}

Conclusion(s) Microfluidic selection yielded spermatozoa with optimal genomic integrity and improved chances of obtaining a euploid conceptus.

Keywords Assisted reproductive technologies $\cdot$ Sperm chromatin fragmentation $\cdot$ Density gradient selection $\cdot$ Microfluidic chamber $\cdot$ ICSI

\section{Introduction}

Infertility affects $12-18 \%$ of couples in the USA of reproductive age [1]. Up to $15 \%$ of all infertility cases may remain unexplained, despite involving couples in which no obvious factor can be traced to either partner [2]. The reproductive treatment approach for these couples is somewhat incremental, beginning with ovulation monitoring in conjunction with

Gianpiero D. Palermo

gdpalerm@med.cornell.edu

1 The Ronald O. Perelman and Claudia Cohen Center for Reproductive Medicine, Weill Cornell Medicine, New York, NY 10021, USA timed intercourse or intrauterine insemination. If these initial treatments fail, a more invasive technique is used such as in vitro fertilization (IVF) or intracytoplasmic sperm injection (ICSI) [3]. When a specific cause for the infertility cannot be identified, a subtle male factor may be presumed. Indeed, up to $11 \%$ of men with a normal semen analysis have a noticeable sperm chromatin fragmentation (SCF), which may affect up to $5 \%$ of men with semen parameters above the 50th percentile $[4,5]$. In addition, an inverse correlation between SCF and sperm motility has been shown [6]; therefore, these men are better suited for ICSI insemination in which sufficiently motile sperm cells are identified under direct visualization [7, 8]. It should be noted that a mild degree of sperm DNA fragmentation can be prevented by ooplasmic repair mechanisms 
[9-11], which have been confirmed by studies assessing the ability of donor eggs to repair spermatozoa with compromised chromatin [12]. Nevertheless, this ooplasmic intervention can be defective in certain couples, particularly in cases where the woman is of advanced reproductive age.

After observing couples with unexplained infertility after a failed IUI, we designed a reproductive treatment algorithm based on a sperm DNA fragmentation assay [13]. According to this algorithm, if the male gamete chromatin integrity is confirmed, the couple can proceed to standard in vitro insemination. Conversely, if an abnormal SCF is reported, the couple is offered ICSI at the outset, utilizing ejaculated spermatozoa. ICSI would also be offered to men who, in spite of having spermatozoa with healthy chromatin, failed to achieve a pregnancy with standard in vitro insemination. Finally, for cases in which a pregnancy has not been achieved even with ICSI, men are counseled by a reproductive urologist and offered a cycle of ICSI using spermatozoa retrieved directly from the testicle $[13,14]$, where SCF appears to be consistently lower $[15,16]$.

Testicular biopsy, however, is a radical and invasive procedure that may result in scarring of the seminiferous tubules with consequent overall impairment of spermatogenesis. Moreover, the correlation between motility, particularly the progressive type, and sperm chromatin integrity prompted us to investigate alternative and more conservative procedures. Among various options, microfluidic devices have been proposed to select more suitable spermatozoa $[15,17-20]$ with higher chromatin integrity and presumably greater fertilizing potential and an improved ability to support embryonic development. Since these novel devices can be sophisticated, expensive, and at times cumbersome, simpler methods have been tested. A recent report has shown the ability, and presumed clinical safety, of a chip sperm sorting device to select directly from the raw semen highly motile spermatozoa characterized by superior chromatin integrity [21].

In this investigation, SCF was assessed on aliquots of sperm specimens selected by a commercial microfluidic sperm sorting device (ZyMōt ${ }^{\mathrm{TM}}$ Multi device; DxNow, Gaithersburg, MD) and standard density gradient in comparison with raw semen. In addition, in couples with recurrent assisted reproductive technology (ART) failure and male partners with high sperm DNA fragmentation, we assessed the fertilization and implantation potential, as well as the ability to support embryonic development, of spermatozoa processed by microfluidic sperm sorting as compared with conventional density gradient centrifugation.

\section{Materials and methods}

\section{Patients}

This study was approved by the Institutional Review Board at Weill Cornell Medicine (IRB No. 1705018205, IRB No.
1210013187, and IRB No. 0712009553), and all couples were counseled and signed informed consent forms in order to participate. From January 2017, a total of 48 patients enrolled in this study. Twenty-three men underwent an initial semen analysis and an additional 25 couples underwent ART cycles.

\section{Semen specimen collection, classification, and selection}

Fresh semen specimens with an average abstinence of $2-$ 5 days were processed for standard semen analysis. Specimens were allowed to liquefy for at least $15 \mathrm{~min}$ at $37^{\circ} \mathrm{C}$ prior to analysis. A small quantity $(5 \mu \mathrm{L})$ of raw sample was loaded onto a Makler chamber. Additional $5-\mu \mathrm{L}$ aliquots of these raw samples were smeared on a morphology slide and evaluated. Volume, concentration, motility, and morphology parameters were appraised according to WHO criteria [2]. Raw specimens were allocated in 2 aliquots for processing by density gradient centrifugation and microfluidic sperm sorting.

Density gradient selection (DGS) After the addition of HEPES-buffered human tubal fluid medium (H-HTF; Irvine Scientific, CA, USA) supplemented with human serum albumin (HSA solution G Series culture media; Vitrolife, Goteborg, Sweden), samples were centrifuged at $600 \mathrm{~g}$ for $10 \mathrm{~min}$. The pellet was loaded onto $1 \mathrm{~mL}$ of $90 \%$ density gradient (Enhance-S Plus Cell Isolation Media, 90\%; Vitrolife), followed by centrifugation at $300 \mathrm{~g}$ for $10 \mathrm{~min}$. Using a glass pipette, the selected sperm pellet was resuspended in medium and centrifuged at $600 \mathrm{~g}$ for $10 \mathrm{~min}$ to remove silica gel particles. The supernatant was discarded, and $5 \mu \mathrm{L}$ of the final sample was reassessed in the Makler chamber for final semen parameters. After preparation, $5 \mu \mathrm{L}$ of the final sample was reassessed in the Makler chamber for the detection of concentration, motility, and morphology.

Microfluidic sperm sorting (MSS) MSS was performed using a commercial single-use device (ZyMōt ${ }^{\mathrm{TM}}$ Multi $(850 \mu \mathrm{L})$ device; DxNow, Gaithersburg, MD). The chip is made of poly(methyl methacrylate) (PMMA) with a single inlet channel that leads to a collection chamber separated by an $8-\mu \mathrm{m}$ porous microfilter that allows spermatozoa with the highest progressive motility to reach the upper outlet chamber for specimen extraction. For ICSI preparation, $850 \mu \mathrm{L}$ of sample was loaded into the inlet of the device. An equivalent amount of HTF medium was loaded on top of the membrane. The loaded device was placed in a humidified incubator at $37{ }^{\circ} \mathrm{C}$ for $30 \mathrm{~min}$. Following incubation, spermatozoa were retrieved using the outlet port with a syringe (Fig. 1). The sorted suspension was reassessed for concentration, motility, morphology, and DNA fragmentation. 


\section{Sperm DNA fragmentation assay}

To assess sperm chromatin integrity, $20 \mu \mathrm{L}$ of sample before and after each selection method was smeared on a slide. Using the samples before and after each selection method, terminal deoxynucleotidyl transferase-mediated deoxyuridine triphosphate-fluorescein nick end labeling (TUNEL) assay protocol was used as previously described [6]. In brief, a commercially available kit was used to perform the assay (in situ cell death detection kit; Roche Diagnostics, Rotkreuz, Switzerland). After production, raw semen samples were allowed to liquefy in an oven at $37^{\circ} \mathrm{C}$ for $15 \mathrm{~min}$. Slides were smeared with $5 \mu \mathrm{L}$ of the semen sample and left to dry overnight. The slides were placed in $4 \%$ paraformaldehyde for $1 \mathrm{~h}$ to allow for fixation of the samples. Slides were then washed in PBS and left to dry overnight once more. Slides were immersed for $2 \mathrm{~min}$ at $4{ }^{\circ} \mathrm{C}$ in a permeabilization solution with $0.1 \%$ Triton $\mathrm{X}-100$ and $0.1 \%$ sodium citrate in PBS. The enzyme and label solutions were applied to the slides according to the dilutions specified in the kit protocol and left to incubate under coverslips in a humidified chamber at $37{ }^{\circ} \mathrm{C}$ for $1 \mathrm{~h}$. Slides were subsequently washed thrice in PBS, and DAPI/Antifade solution was added in order to visualize sperm nuclei, which were observed under a fluorescent microscope for signals indicating DNA breakage. A minimum of 500 spermatozoa was assessed per patient; a sperm DNA fragmentation (SDF) of $\leq 15 \%$ was considered normal.

\section{Ovarian superovulation and oocyte collection}

Only couples with normal BMI and no history of smoking, excess drinking, or use of recreational drugs were included. All patients included were Caucasian and had comparable durations and indications of infertility. The average AMH of the female partner was $3.2 \pm 3 \mathrm{ng} / \mathrm{mL}$, the average FSH was $5.3 \pm 4 \mathrm{IU} / \mathrm{mL}$, and the average peak estradiol for ICSI stimulation was $3066.3 \pm 1731 \mathrm{pg} / \mathrm{mL}$.

A similar superovulation protocol was used for all couples. Patients were treated with daily gonadotropins (Follistim; Merck, Kenilworth, NJ, USA; Gonal-F; EMD-Serono, Geneva, Switzerland; and/or Menopur; Ferring Pharmaceuticals Inc, Parsippany, NJ, USA). Precocious ovulation was prevented by GnRH antagonist administration (Ganirelix acetate; Merck, Kenilworth, NJ, USA; or Cetrotide; EMD-Serono Inc., Rockland, MA, USA). The trigger for final oocyte maturation with human chorionic gonadotropin (Pregnyl, Merck) was administered when the two leading follicles reached a diameter of $\geq$ $17 \mathrm{~mm}[22,23]$. Transvaginal oocyte retrieval was performed under conscious sedation 35-37 h after hCG administration.

Cumulus-corona cells were removed by exposure to medium containing $40 \mathrm{IU} / \mathrm{mL}$ hyaluronidase (Cumulase; Halozyme Therapeutics, Inc., San Diego, CA) [24] and incubated $1-2 \mathrm{~h}$ prior to ICSI.

\section{Embryo culture and morphologic and cytogenetic evaluation}

Embryo evaluation, criteria, and the biopsy procedure have been described previously [25]. Day-3 embryos considered good quality had morphologic grades of $1-2$ ( $\geq 8$ cells with even or slightly uneven blastomere expansion and $\leq 20 \%$ fragmentation). Day-5 good-quality embryos had the following morphologic grades: blastocele, 1-3 (degree of expansion $\geq$ $50 \%$ the volume of the embryo); inner cell mass, A-B (clear inner cell mass with healthy cells); and trophectoderm, A-B (healthy cells).

The biopsy procedure was performed on day 5 as follows: The embryo was positioned by a holding a pipette while laser pulses (ZI-LOS-tk Laser) were used to create an opening in the zona pellucida to allow for aspiration of three to seven trophoblastic cells with a $20-\mu \mathrm{m}$-diameter biopsy pipette. Cells were transferred in $200 \mu \mathrm{L}$ of polymerase chain reaction tubes with $2 \mu \mathrm{L}$ of lysis buffer and analyzed by array comparative genome hybridization (Bluegnome 24SureV3 chip; Illumina, San Diego, CA). Biopsied embryos were rinsed in a culture buffer and then vitrified [26] until the cytogenetic results were available and patient synchronization was achieved for embryo transfer.

\section{Embryo transfer and pregnancy assessment}

Patients received intramuscular progesterone supplementation (50 mg daily) starting 1 day after retrieval. Couples underwent a fresh embryo transfer on day 3 or 5 according to the developmental characteristics of the embryo. Couples that underwent preimplantation genetic testing for aneuploidy (PGT-A) had euploid embryo transfers in natural or programmed FET cycles [27]. Endometrial lining was at least $7 \mathrm{~mm}$ in order for a patient to be considered for an embryo transfer. Serum $\beta$ hCG levels were measured 14 days after retrieval. Clinical pregnancy was defined as the detection of an intrauterine implantation sac with fetal heart activity on ultrasound.

\section{Statistical analysis}

Continuous variables were described as a mean $\pm \mathrm{SD}$ (standard deviation). The Friedman test was used when the data was paired and had 3 or more treatment groups. The KruskalWallis test was used when there were 3 or more treatment groups, but the data was unpaired. The Mann-Whitney test was used when data was unpaired and two treatments were assessed at a time. Variables in this category include volume, concentration, motility, normal forms, and DNA fragmentation. For categorical variables, represented as a percentage, the chi-square test was performed to test for relationships between the two groups. Variables in this category are fertilization, 
Table 1 Parameters and SCF values in aliquots of specimen processed by density gradient and microfluidics in comparison to raw semen of 23 men

\begin{tabular}{|c|c|c|c|c|}
\hline \multirow[t]{2}{*}{ Parameters (mean $\pm \mathrm{SD}$ ) } & \multicolumn{4}{|l|}{ Selection } \\
\hline & Raw & Density gradient & Microfluidics & $P$ value \\
\hline Volume (mL) & $2.9 \pm 1.7 *^{\dagger}$ & $0.5 \pm 0.02 *$ & $0.4 \pm 0.08^{\dagger}$ & $<0.0001$ \\
\hline Concentration $\left(\times 10^{6} / \mathrm{mL}\right)$ & $61.8 \pm 35^{* \dagger}$ & $40.5 \pm 25^{*}$ & $15.3 \pm 12^{\dagger \ddagger}$ & $<0.0001$ \\
\hline Motility (\%) & $34.1 \pm 14^{* \dagger}$ & $65.4 \pm 31.4^{* *}$ & $96 \pm 11^{\dagger+}$ & $<0.0001$ \\
\hline Progressive (\%) & $30.1 \pm 14^{* \dagger}$ & $67.2 \pm 29.7 * *$ & $97.6 \pm 2^{\dagger *}$ & $<0.0001$ \\
\hline Non-progressive (\%) & $4.3 \pm 2 * \dagger$ & $2.1 \pm 1.5^{*}$ & $0.08 \pm 0.2^{\dagger \dagger}$ & $<0.0001$ \\
\hline Morphology (\%) & $2.5 \pm 0.8^{\dagger}$ & $2.6 \pm 1^{\ddagger}$ & $4.0 \pm 0.7^{\dagger *}$ & $<0.0001$ \\
\hline DNA fragmentation (\%) & $20.7 \pm 10^{* \dagger}$ & $12.5 \pm 5^{* \ddagger}$ & $1.8 \pm 1^{\dagger+}$ & $<0.0001$ \\
\hline
\end{tabular}

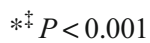

${ }^{\dagger} P<0.0002$

This table describes the semen characteristics (including the DNA fragmentation) of the raw specimen and compared them with those obtained after density gradient and microfluidic selection. Statistical evaluation is depicted comparing the values between raw specimen and each selection method clinical pregnancy, and pregnancy loss. A $P$ value was reported when the differences between the groups were statistically significant (at a threshold of 0.05). R software (R Foundation for Statistical Computing; Vienna, Austria) was used to perform all statistical analyses.

\section{Results}

To test the efficacy of this novel selection method, particularly its ability to identify the highest progressively motile spermatozoa characterized by optimal chromatin integrity, we screened 48 men for SCF levels using raw semen, and after processing by DGS and MSS. Semen specimens were obtained from 23 men and SCF was assessed following 3 aliquots from each specimen: raw, density gradient, and microfluidic processing. Although the sperm concentration following MSS decreased $(P<0.0001)$, motility and normal morphology significantly improved $(P<0.0001)$. Furthermore, sperm DNA fragmentation decreased from $20.7 \%$ in the raw specimen to $1.8 \%$ after MSS $(P<0.0001$; Table 1 and Fig. 2$)$.
Encouraged by these findings, we then applied the same method to couples who underwent ICSI. This cohort included 25 couples (maternal age, $37.3 \pm 6$ years; paternal age, $44.4 \pm$ 10 years). The superovulation protocol of the female partners and the couples' age, BMI, history of infertility, ethnicity, indication to infertility, smoking and drinking habits, and the use of recreational drugs were comparable or controlled for among these 25 patients.

Semen characteristics and SCF were evaluated before and after DGS and MSS processing for each patient, who therefore served as their own control (Table 2). While there was a decrease in concentration $(P<0.0001)$, a concurrent improvement in total motility, progressive motility, and morphology of the microfluidic sorted aliquots was seen $(P<0.0001)$. SCF dropped below threshold after density gradient selection $(P<0.0001)$ and fell dramatically after microfluidic sorting $(P<0.0001$; Table 2).

Next, to compare the impact of selected spermatozoa on embryo developmental competence, we treated four couples with male partners who had a remarkably elevated SCF in their ejaculate $(34.1 \%)$. The semen parameters for microfluidics as

\section{Sperm Collection}

Fig. 1 Raw semen samples are loaded into the bottom chamber of the microfluidic device through an inlet and incubated. Spermatozoa with the highest progressive motility and superior chromatin integrity are able to pass through the porous membrane into the upper chamber, where they are collected after incubation

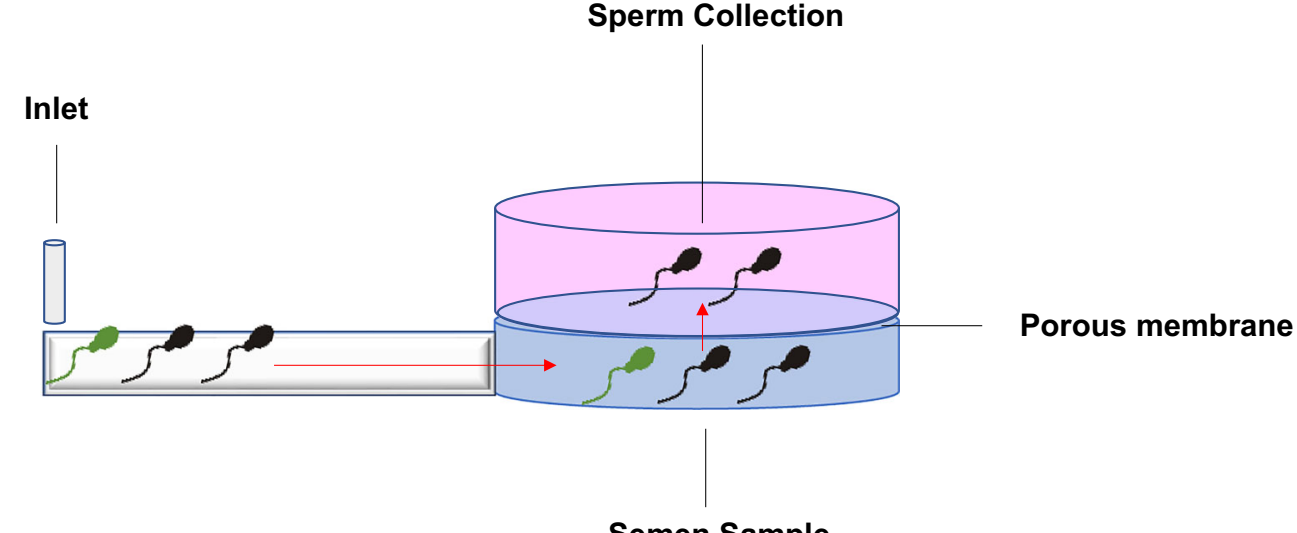

Semen Sample 
Table 2 Parameters and SCF values in aliquots of specimen processed by density gradient and microfluidics in comparison to raw semen of the male partner of 25 couples undergoing ICSI

\begin{tabular}{|c|c|c|c|c|}
\hline \multirow[t]{2}{*}{ Parameters (mean $\pm \mathrm{SD}$ ) } & \multicolumn{3}{|l|}{ Selection } & \multirow[t]{2}{*}{$P$ value } \\
\hline & Raw & Density gradient & Microfluidics & \\
\hline Volume (mL) & $1.9 \pm 1.0^{* \dagger}$ & $0.5 \pm 0.1 * \ddagger$ & $0.4 \pm 0.05^{\dagger \dagger}$ & $<0.0001$ \\
\hline Concentration $\left(\times 10^{6} / \mathrm{mL}\right)$ & $32.7 \pm 34 * \dagger$ & $25.8 \pm 30^{* \star}$ & $7.5 \pm 11^{\dagger *}$ & $<0.0001$ \\
\hline Motility (\%) & $30.2 \pm 13 * \dagger$ & $53.1 \pm 30^{* \star}$ & $98 \pm 3^{\dagger+}$ & $<0.0001$ \\
\hline Progressive $(\%)$ & $26.0 \pm 13^{* \dagger}$ & $51.2 \pm 31$ *末 & $97.7 \pm 3^{\dagger *}$ & $<0.0001$ \\
\hline Non-progressive (\%) & $4.1 \pm 3 *^{\dagger}$ & $1.8 \pm 2.2^{*}$ & $0.2 \pm 0.8^{\dagger *}$ & $<0.0001$ \\
\hline Morphology (\%) & $2.1 \pm 0.7^{\dagger}$ & $2.3 \pm 1^{*}$ & $3 \pm 1^{\dagger \dagger}$ & $<0.0001$ \\
\hline DNA fragmentation (\%) & $28.8 \pm 9 *^{\dagger}$ & $21 \pm 9 * *$ & $1.3 \pm 0.7^{\dagger *}$ & $<0.0001$ \\
\hline
\end{tabular}

$* \hbar+P<0.05$

${ }^{\dagger} P<0.001$

This table describes the semen characteristics (including the DNA fragmentation) of the raw specimen and compared them with those obtained after density gradient and microfluidic selection. Statistical evaluation is depicted comparing the values between raw specimen and each selection method well as the SCF are depicted in Table 3. In this group of men, chromatin integrity improved dramatically $(1.6 \%)$ when spermatozoa went through the microfluidic chamber $(P<0.02)$ (Fig. 1). There were also significant improvements in motility $(P<0.02)$, with a minor improvement in spermatozoa morphology. These couples (maternal age, $35.9 \pm 4$ years; paternal age, $40.0 \pm 6$ years) underwent 11 ICSI cycles with DGS and achieved $26.3 \%$ good-quality embryos with a fertilization rate of $59 \%$ that yielded a biochemical pregnancy and a pregnancy loss at 7 weeks. Once MSS spermatozoa were used in four cycles, a comparable fertilization rate $(61.2 \%)$ was achieved with a trend toward a larger proportion of good-quality embryos $(57.1 \%)$ that resulted in an implantation rate of $25 \%$ and a clinical pregnancy rate of $50 \%$ (Table 4 ).

We then included 5 couples (maternal age, $36.4 \pm 5$ years; paternal age, $36.7 \pm 7$ years) who planned to have their embryos screened by PGT-A, following ICSI. When sperm samples from the male partners in this group were processed by DGC, the motility significantly improved (28\% raw vs $51 \%$ DGS; $P<0.05)$, while sperm morphology and SCF remained comparable. After using MSS, there was a clear and net improvement in motility (28\% raw vs 99\% MSS; $P<0.0001)$ and SCF $(19.2 \%$ raw vs $1.5 \%$ MSS; $P<0.01)$ (Table 5). There was also a reduction in concentration, but this was not detrimental to ICSI insemination. These couples underwent 12 ICSI cycles where semen specimens were processed by DGS, resulting in a satisfactory fertilization rate $(78 \%)$ and an adequate number $(23 / 33,69.7 \%)$ of morphologically good-quality embryos. PGT-A identified 10 euploid embryos that generated a pregnancy, which did not go to term. In the 9 cycles in which MSS was used, there was a comparable fertilization rate $(72.8 \%)$ as well as number $(27 / 38,71 \%)$ of good-quality embryos of which half were confirmed euploid. Thus far, 4 embryos were transferred in four couples, all of which implanted and resulted in ongoing clinical pregnancies $(P<0.01$; Table 6$)$.

Finally, 16 couples that had been treated at other clinics and failed to achieve a pregnancy underwent ICSI solely with MSS at our center. In a preliminary comparison of semen parameters following DGS and MSS, motility (59.3\% DGS vs $97.6 \%$ MSS; $P<0.02)$ and morphology $(2.1 \%$ DGS vs $3.3 \%$ MSS; $P<0.05)$ increased dramatically while SCF $(18.5 \%$ DGS vs
Table 3 Semen parameters and SCF of couples that underwent ICSI by DGS processing and subsequently by MSS

\begin{tabular}{lllll}
\hline & \multicolumn{2}{l}{ Selection } & & $P$ value \\
\cline { 2 - 4 } & Raw & Density gradient* & Microfluidics & \\
\hline Volume $(\mathrm{mL})$ & $1.4 \pm 2$ & $0.2 \pm 0.1$ & $0.4 \pm 0.1$ & $<0.05$ \\
Concentration $\left(\times 10^{6} / \mathrm{mL}\right)$ & $2.0 \pm 5$ & $3.1 \pm 5$ & $0.12 \pm 0.1$ & $<0.05$ \\
Motility $(\%)$ & $3.6 \pm 12$ & $10 \pm 13$ & $96.5 \pm 5$ & $<0.02$ \\
Morphology $(\%)$ & $1.2 \pm 0.5$ & $1.4 \pm 0.5$ & $2.0 \pm 0$ & $\mathrm{NS}$ \\
DNA fragmentation $(\%)$ & $34.1 \pm 9$ & $26 \pm 4$ & $1.6 \pm 0.7$ & $<0.02$ \\
\hline
\end{tabular}

*3 samples were processed with centrifugation method due to low concentration

This table describes the semen characteristics (including the DNA fragmentation) of the raw specimen and compared them with those obtained after density gradient and microfluidic selection. Statistical evaluation is depicted comparing the values between raw specimen and each selection method 


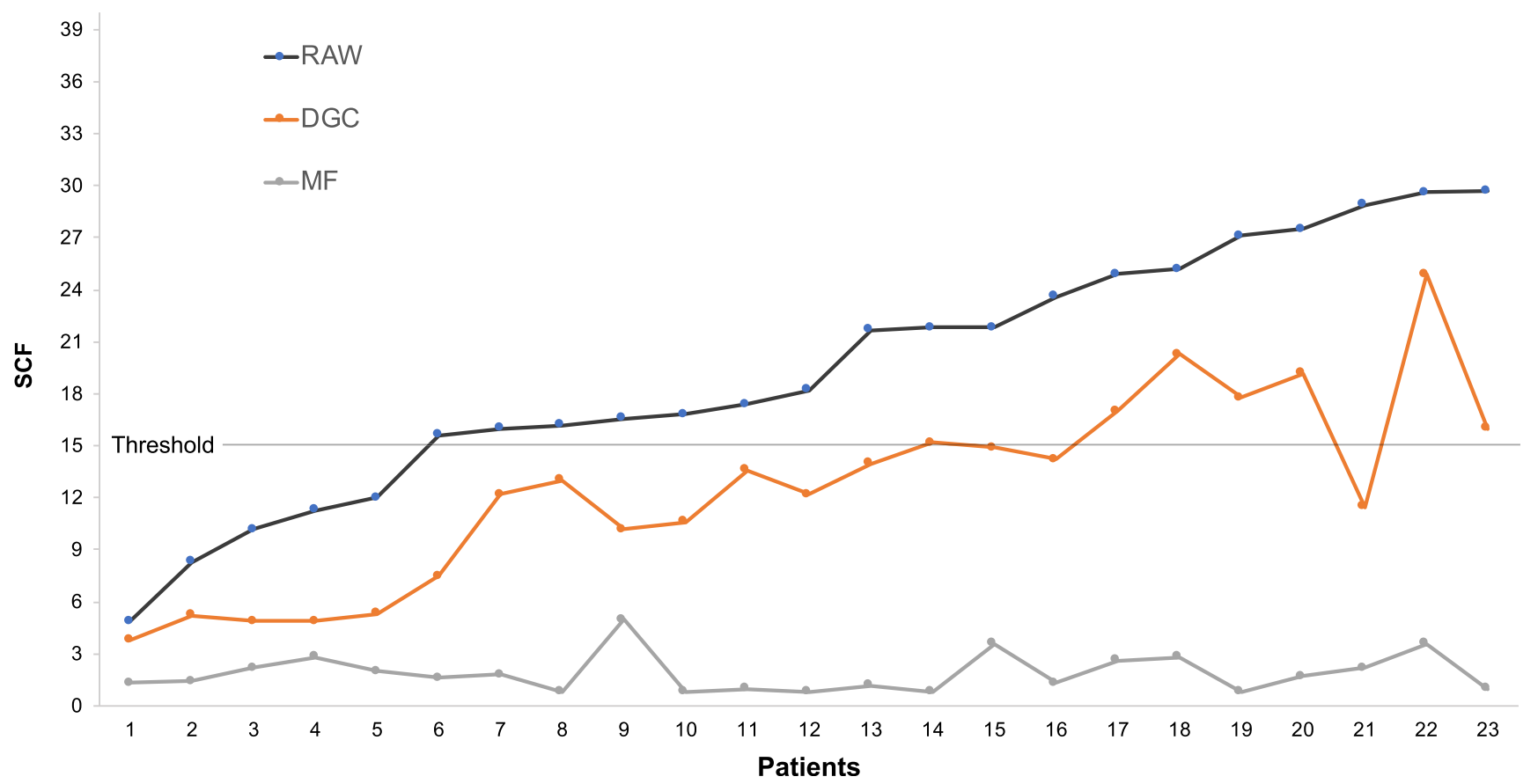

Fig. 2 Ranked SCF assessment carried out in 23 men and compared to the level of chromatin integrity achieved after DGS and MSS

$1.2 \%$ MSS; $P<0.02$ ) decreased (Table 7). Of these couples, 8 underwent 13 ICSI cycles elsewhere, yielding a fertilization rate of $74.1 \%$, but no pregnancies were reported. A total of 9 couples underwent 12 ICSI cycles with MSS and fresh embryo transfer, with a $74 \%$ fertilization rate and $83.3 \%(20 / 24)$ good-quality embryos. These cycles resulted in $25 \%(6 / 24)$ implantation and $50 \%(6 / 12 ; P<0.05)$ clinical pregnancy rate

Table 4 Clinical outcome of couples that underwent ICSI by DGS processing and subsequently by MSS

\begin{tabular}{|c|c|c|c|}
\hline \multirow[t]{2}{*}{ Number of $(\%)$} & \multicolumn{2}{|l|}{ Selection } & \multirow[t]{2}{*}{$P$ value } \\
\hline & Density gradient & Microfluidics & \\
\hline Patients & 4 & & \\
\hline Cycles & 11 & 4 & \\
\hline Injected oocytes $(M \pm \mathrm{SD})$ & $7.5 \pm 5$ & $12.2 \pm 6$ & NS \\
\hline Fertilization rate (2PN) & $49 / 83(59.0)$ & $30 / 49(61.2)$ & NS \\
\hline Embryo transfers & 4 & 4 & \\
\hline Embryos transferred & 19 & 8 & \\
\hline Good quality & $5 / 19(26.3)$ & $4 / 7(57.1)$ & NS \\
\hline \multicolumn{4}{|l|}{ Pregnancy with } \\
\hline$+\beta h c g$ & $2 / 4(50.0)$ & $2 / 4(50.0)$ & NS \\
\hline$+\mathrm{FHB}$ & $1 / 4(25.0)$ & $2 / 4(50.0)$ & NS \\
\hline Implantation & $1 / 19(5.2)$ & $2 / 8(25.0)$ & NS \\
\hline Clinical pregnancy rate & $1 / 4(25.0)$ & $2 / 4(50.0)$ & NS \\
\hline Pregnancy loss & $1 / 1(100.0)$ & $0 / 4(0.0)$ & NS \\
\hline Ongoing/delivered & $0 / 4(0.0)$ & $2 / 4(50.0)$ & NS \\
\hline
\end{tabular}

This table describes the clinical outcome of 4 couples that underwent ICSI with spermatozoa processed by density gradient and later by microfluidics. Statistical analysis is presented
(Table 8). The remaining 7 couples who underwent 8 ICSI cycles with MSS opted for PGT-A due to their history of having all chromosomally abnormal embryos. In these couples, a fertilization rate of $78.4 \%(62 / 79)$ progressed to $62.1 \%(23 / 79)$ good-quality embryos of which $51 \%(19 / 37 ; P<0.001)$ were euploid. Five embryos were transferred in four patients who achieved an $80 \%(4 / 5 ; P<0.01)$ implantation and clinical pregnancy rate $(P=0.01)$ (Table 8$)$.

\section{Discussion}

Unexplained infertility may be attributed to a subtle male factor linked to compromised sperm DNA integrity. During ICSI, it is possible to select the most progressively motile spermatozoa that therefore have the highest chromatin integrity [6]. Moreover, it has been known for some time that once the male genome penetrates the oocyte, ooplasmic mechanisms decondense sperm chromatin and reconstitute nucleosomes by replacing protamine with histones while processing and repairing DNA strands $[9,10,28,29]$. Therefore, a young oocyte along with the selection of the most motile spermatozoa for ICSI insemination may prevent these male genomic defects $[10,11,29]$.

In the most persistent cases of male factor infertility, utilizing spermatozoa retrieved directly from the testis, presumed to retain superior chromatin integrity, has been proposed [13, 14 , 30]. However, this technique is not without drawbacks, as gametes retrieved directly from the seminiferous tubules can have lower fertilization capacity, which is typical of testicular 
Table 5 Parameters of SCF of sperm specimen processed by density gradient or microfluidics of couples undergoing ICSI

\begin{tabular}{lllll}
\hline Parameters (mean \pm SD) & \multicolumn{2}{l}{ Selection } & \multirow{2}{*}{$P$ value } \\
\cline { 2 - 4 } & Raw & Density gradient & Microfluidics & \\
\hline Volume $(\mathrm{mL})$ & $2.2 \pm 1.7^{* \dagger}$ & $0.5 \pm 0.4^{*}$ & $0.5 \pm 0.2^{\dagger}$ & $<0.001$ \\
Concentration $\left(\times 10^{6} / \mathrm{mL}\right)$ & $38 \pm 42$ & $2.6 \pm 2$ & $2.8 \pm 4.4$ & $\mathrm{NS}$ \\
Motility $(\%)$ & $28 \pm 22^{*^{\dagger}}$ & $51 \pm 30^{* \ddagger}$ & $99 \pm 1^{\dagger \dagger}$ & $<0.0001$ \\
Morphology $(\%)$ & $2.6 \pm 0.9$ & $3.0 \pm 1$ & $3.8 \pm 0.8$ & $\mathrm{NS}$ \\
DNA fragmentation $(\%)$ & $19.2 \pm 5^{\dagger}$ & $14.2 \pm 4^{\dagger}$ & $1.5 \pm 1.4^{\dagger \dagger}$ & $<0.01$ \\
\hline
\end{tabular}

$* P<0.05$

$\dagger P<0.02$

This table describes the semen characteristics (including the DNA fragmentation) of the raw specimen and compared them with those obtained after density gradient and microfluidic selection. Statistical evaluation is depicted comparing the values between raw specimen and each selection method spermatozoa. Moreover, microsurgical testicular biopsy, although focused on individual tubules, is an invasive and costly procedure that may be associated with damage of the surrounding tissue with consequent scarring, requiring approximately 6 months to heal [31].

These complications led us to investigate alternative methods of identifying spermatozoa with a less impaired genome [32-34]. Indeed, this can be achieved to some extent with a standard sperm selection technique such as DGS, which appears to lower the level of SCF [35]. This well-established method, however, does not lower SCF below the normal threshold due to its centrifugation steps that generate reactive oxygen species (ROS) and its inability to select the most progressively motile spermatozoa [36-38]. Therefore, the known direct relationship between sperm progressive motility and chromatin integrity persuaded us to test a novel device that selects the most motile spermatozoa without having to centrifuge the specimen (Tables 1 and 2).

Our initial goal was to find a way to treat couples that had an impairment in male genomic integrity and a prior history of ART failure without resorting to invasive surgical techniques. The enhancement of motility, progression, and morphology we observed with the use of MSS was encouraging, but it was not as important as the remarkable reduction to SCF (Table 1), as has been confirmed by others [21]. These findings encouraged us to test this method of sperm sorting on 25 couples struggling with compromised SCF and persistent ART failure. The same effects of MSS on semen parameters were seen when preparing these semen samples for ICSI treatment. Intriguingly, we were able to achieve an average SCF
Table 6 Clinical outcome of couples who underwent ICSI with DGS and subsequently MSS and received a thawed PGT-A screen embryo transfer

\begin{tabular}{|c|c|c|c|}
\hline \multirow[t]{2}{*}{ Number of $(\%)$} & \multicolumn{2}{|l|}{ Selection } & \multirow[t]{2}{*}{$P$ value } \\
\hline & Density gradient & Microfluidics & \\
\hline Patient & 5 & & \\
\hline Cycles & 12 & 9 & \\
\hline Injected oocytes $(M \pm \mathrm{SD})$ & $8.1 \pm 4$ & $9 \pm 4$ & NS \\
\hline Fertilization rate $(2 \mathrm{PN})(\%)$ & $76 / 97(78 \%)$ & $59 / 81(72.8 \%)$ & NS \\
\hline Embryos screened & 33 & 38 & \\
\hline Good quality & $23 / 33(69.7 \%)$ & $27 / 38(71.0 \%)$ & NS \\
\hline Euploid & $10 / 33(30.3 \%)$ & $12 / 38(31.5 \%)$ & NS \\
\hline Embryos transferred after PGT-A & 10 & 4 & NS \\
\hline \multicolumn{4}{|l|}{ Pregnancy with } \\
\hline$+\beta$ hcg $(\%)$ & $1 / 5(20 \%)$ & $4 / 4(100 \%)$ & $<0.05$ \\
\hline$+\mathrm{FhB}$ & $1 / 5(20 \%)$ & $4 / 4(100 \%)$ & $<0.05$ \\
\hline Implantation & $1 / 10(10 \%)$ & $4 / 4(100 \%)$ & $<0.01$ \\
\hline Clinical pregnancy rate & $1 / 5(20 \%)$ & $4 / 4(100 \%)$ & $<0.05$ \\
\hline Pregnancy loss & $1 / 1(100 \%)$ & $0 / 4(0 \%)$ & NS \\
\hline Ongoing/delivered (\%) & $0 / 5(0 \%)$ & $4 / 4(100 \%)$ & $<0.01$ \\
\hline
\end{tabular}

This table describes the clinical outcome after PGT-A of 5 couples that underwent ICSI with spermatozoa processed by density gradient and later by microfluidics. Statistical analysis is presented 
Table 7 Parameters of SCF of sperm specimen of 16 couples treated solely by microfluidic sperm selection at our center

\begin{tabular}{lllll}
\hline & \multicolumn{2}{l}{ Selection } & & $P$ value \\
\cline { 2 - 4 } & Raw & Density gradient & Microfluidics & \\
\hline Volume $(\mathrm{mL})$ & $1.8 \pm 1^{*^{\dagger}}$ & $0.5 \pm 0.02^{*}$ & $0.5 \pm 0.05^{\dagger}$ & $<0.05$ \\
Concentration $\left(\times 10^{6}\right)$ & $40 \pm 39^{\dagger}$ & $32.3 \pm 36^{\ddagger}$ & $9.1 \pm 13^{\dagger \dagger}$ & $<0.05$ \\
Motility $(\%)$ & $32 \pm 12^{*^{\dagger}}$ & $59.3 \pm 29^{*}$ & $97.6 \pm 2^{\dagger \dagger}$ & $<0.02$ \\
Morphology $(\%)$ & $2.1 \pm 1^{\dagger}$ & $2.1 \pm 1^{\dagger}$ & $3.3 \pm 1.1^{\dagger \dagger}$ & $<0.05$ \\
DNA fragmentation \%) & $29 \pm 9^{* \dagger}$ & $18.5 \pm 11^{* \dagger}$ & $1.2 \pm 0.4^{\dagger \dagger}$ & $<0.02$ \\
\hline
\end{tabular}

$* \pm+P<0.05$

${ }^{\dagger} P<0.005$

This table describes the semen characteristics (including the DNA fragmentation) of the raw specimen and compared them with those obtained after density gradient and microfluidic selection. Statistical evaluation is depicted comparing the values between raw specimen and each selection method lower than that of the 23 men we tested initially, even though this group of 25 had higher SCF compared with those men (Table 2).

In couples who had a fresh embryo replacement, MSS yielded spermatozoa with higher genomic competence as demonstrated by their ability to establish healthy pregnancies compared with cycles in which spermatozoa were processed in a standard fashion (Table 4). This was also the case for other groups that had spermatozoa selected using an apoptotic marker [39]. Interestingly, this was even true for couples who underwent PGT-A, as confirmed by the higher portion of euploid embryos that resulted in no term pregnancies (Table 6). In this cohort, MSS yielded a comparable proportion of euploid embryos and, for those couples who received a transfer by the time of this publication, all resulted in a viable pregnancy (Table 6). This remained true when we treated 16 couples with a history of recurrent implantation failure who, once treated by MSS, reported a clinical pregnancy rate of $58.8 \%$ (Table 8).

From these observations, it appears that a phenotypic selection of spermatozoa with the highest motility may yield a greater number of euploid embryos capable of

Table 8 Reproductive history of 16 couples treated solely by microfluidic sperm selection at our center

\begin{tabular}{|c|c|c|c|c|c|}
\hline & \multicolumn{5}{|l|}{ Microfluidics } \\
\hline & DGS elsewhere $^{\mathrm{a}}$ & Fresh embryo transfer ${ }^{\mathrm{b}}$ & PGT-A embryo transfer ${ }^{c}$ & $P$ value $\mathrm{e}^{\mathrm{a}, \mathrm{b}}$ & $P$ value $\mathrm{a}^{\mathrm{a}, \mathrm{c}}$ \\
\hline Patients & 8 & 9 & 7 & & \\
\hline Cycles & 13 & 12 & 8 & & \\
\hline Injected oocytes $(M \pm \mathrm{SD})$ & $8.3 \pm 9$ & $10 \pm 8$ & $8.7 \pm 5$ & NS & NS \\
\hline Injected oocytes & 116 & 123 & 79 & & \\
\hline Fertilization rate $(2 \mathrm{PN})$ & $86 / 116(74.1 \%)$ & $91 / 123(74 \%)$ & $62 / 79(78.4 \%)$ & NS & NS \\
\hline Embryos screened & 12 & - & 37 & & \\
\hline Good quality & N/A & $20 / 24(83.3 \%)$ & $23 / 37(62.1 \%)$ & & \\
\hline Euploid & $0 / 12(0 \%)$ & - & $19 / 37(51 \%)$ & - & $<0.001$ \\
\hline Cycle w/ transfer & 7 & 12 & 5 & & \\
\hline Embryos transferred & 8 & 24 & 5 & & \\
\hline \multicolumn{6}{|l|}{ Pregnancy with } \\
\hline$+\beta$ hcg & $0 / 7(0 \%)$ & $7 / 12(58.3 \%)$ & $4 / 5(80 \%)$ & $<0.05$ & 0.01 \\
\hline$+\mathrm{FhB}$ & $0 / 7(0 \%)$ & $6 / 12(50 \%)$ & $4 / 5(80 \%)$ & $<0.05$ & 0.01 \\
\hline Implantation & $0 / 8(0 \%)$ & $6 / 24(25 \%)$ & $4 / 5(80 \%)$ & NS & $<0.01$ \\
\hline Clinical pregnancy rate & $0 / 7(0 \%)$ & $6 / 12(50 \%)$ & $4 / 5(80 \%)$ & $<0.05$ & 0.01 \\
\hline Pregnancy loss & - & $2 / 6(33 \%)$ & $0 / 4(0 \%)$ & - & - \\
\hline Ongoing/delivered & $0 / 6(0 \%)$ & $4 / 12(33 \%)$ & $4 / 5(80 \%)$ & NS & 0.01 \\
\hline
\end{tabular}

This table describes the clinical outcome of couples that underwent ICSI at our center with spermatozoa processed by microfluidics. Results are distinguished between couples that underwent a fresh embryo transfer or with PGT-A selection. Historical cycles performed elsewhere with density gradient selection are also reported for comparison. Statistical analysis is presented 
resulting in a viable pregnancy. While it is difficult to comprehend how selecting spermatozoa with higher chromatin integrity would have any impact on the number of euploid embryos generated, this can only be explained by the type of nicks and breaks present within the chromatin $[10,28,40]$. When an SCF assessment was carried out with an alkaline Comet assay to detect single-strand DNA breaks and a neutral Comet assay to identify double-strand DNA breaks, the frequency of these chromatin defects was equivalent at about $50 \%$ in the fertile control as well as in oligo-astheno-teratozoospermic patients [41]. Indeed, double-strand breaks may actually affect the chromosomal integrity of the male genome with consequent contribution to embryo aneuploidy, as has been seen in couples with idiopathic and recurrent pregnancy losses [42]. However, this will require further research utilizing an assay capable of specifically detecting this type of DNA damage, such as the neutral Comet assay.

\section{Conclusions}

Within the quest for an optimal spermatozoon to inject during ICSI, this study proposes a non-invasive method to recover spermatozoa with superior genomic competence for ICSI treatment in men with high SCF in their ejaculate. These findings allow us to reconsider the relevant contribution of the male genome to the normal development of the conceptus. Most importantly, this study proposes a conservative approach to treat couples with recurrent ART and/or implantation failure without resorting to testicular biopsy.

Acknowledgements We would like to thank Julie Novak for her professional statistical analysis of our data. We would also like to thank Alexandra MacWade for her help in editing the manuscript. Finally, we are especially grateful to Aysha Trout for her help in gathering the data.

\section{Compliance with ethical standards}

This study was approved by the Institutional Review Board at Weill Cornell Medicine (IRB No. 1705018205, IRB No. 1210013187, and IRB No. 0712009553), and all couples were counseled and signed informed consent forms in order to participate.

Open Access This article is distributed under the terms of the Creative Commons Attribution 4.0 International License (http:// creativecommons.org/licenses/by/4.0/), which permits unrestricted use, distribution, and reproduction in any medium, provided you give appropriate credit to the original author(s) and the source, provide a link to the Creative Commons license, and indicate if changes were made.

\section{References}

1. Thoma ME, McLain AC, Louis JF, King RB, Trumble AC, Sundaram R, et al. Prevalence of infertility in the United States as estimated by the current duration approach and a traditional constructed approach. Fertil Steril. 2013;99(5):1324-31.e1. https://doi. org/10.1016/j.fertnstert.2012.11.037.

2. WHO laboratory manual for the examination and processing of human semen. 5th ed. ed. Geneva : World Health Organization; 2010.

3. Intracytoplasmic sperm injection (ICSI) in 2006: evidence and evolution. Human reproduction update. 2007;13(6):515-26. doi: https://doi.org/10.1093/humupd/dmm024.

4. Zini A, Fischer MA, Sharir S, Shayegan B, Phang D, Jarvi K. Prevalence of abnormal sperm DNA denaturation in fertile and infertile men. Urology. 2002;60(6):1069-72.

5. Belloc S, Hazout A, Zini A, Merviel P, Cabry R, Chahine H, et al. How to overcome male infertility after 40 : influence of paternal age on fertility. Maturitas. 2014;78(1):22-9. https://doi.org/10.1016/j. maturitas.2014.02.011.

6. Palermo GD, Neri QV, Cozzubbo T, Rosenwaks Z. Perspectives on the assessment of human sperm chromatin integrity. Fertil Steril. 2014;102(6):1508-17. https://doi.org/10.1016/j.fertnstert.2014.10. 008.

7. Simon L, Proutski I, Stevenson M, Jennings D, McManus J, Lutton $\mathrm{D}$, et al. Sperm DNA damage has a negative association with livebirth rates after IVF. Reprod BioMed Online. 2013;26(1):68-78. https://doi.org/10.1016/j.rbmo.2012.09.019.

8. Zeyad A, Hamad M, Amor H, Hammadeh ME. Relationships between bacteriospermia, DNA integrity, nuclear protamine alteration, sperm quality and ICSI outcome. Reprod Biol. 2018;18(1): 115-21. https://doi.org/10.1016/j.repbio.2018.01.010.

9. Ashwood-Smith MJ, Edwards RG. DNA repair by oocytes. Mol Hum Reprod. 1996;2(1):46-51.

10. Menezo Y, Dale B, Cohen M. DNA damage and repair in human oocytes and embryos: a review. Zygote. 2010;18(4):357-65. https:// doi.org/10.1017/s0967199410000286.

11. Sakkas D, Alvarez JG. Sperm DNA fragmentation: mechanisms of origin, impact on reproductive outcome, and analysis. Fertil Steril. 2010;93(4):1027-36. https://doi.org/10.1016/j.fertnstert.2009.10. 046.

12. Humm KC, Sakkas D. Role of increased male age in IVF and egg donation: is sperm DNA fragmentation responsible? Fertil Steril. 2013;99(1):30-6. https://doi.org/10.1016/j.fertnstert.2012.11.024.

13. O'Neill CL, Parrella A, Keating D, Cheung S, Rosenwaks Z, Palermo GD. A treatment algorithm for couples with unexplained infertility based on sperm chromatin assessment. J Assist Reprod Genet. 2018;35(10):1911-7. https://doi.org/10.1007/s10815-0181270-x.

14. Greco E, Scarselli F, Iacobelli M, Rienzi L, Ubaldi F, Ferrero S, et al. Efficient treatment of infertility due to sperm DNA damage by ICSI with testicular spermatozoa. Hum Reprod. 2005;20(1):22630. https://doi.org/10.1093/humrep/deh590.

15. Esteves SC. Testicular versus ejaculated sperm should be used for intracytoplasmic sperm injection (ICSI) in cases of infertility associated with sperm DNA fragmentation | Opinion: Yes. Int braz $\mathrm{j}$ Urol : Off J Braz Soc Urol. 2018;44(4):667-75. https://doi.org/10. 1590/S1677-5538.IBJU.2018.04.03.

16. Parrella A, O’Neill C, Chow S, Goldstein M, Rosenwaks Z, Palermo GD. Sperm source influences the extent of DNA fragmentation and shapes reproductive outcome. Fertil Steril. 2017;108(3): e309. https://doi.org/10.1016/j.fertnstert.2017.07.914.

17. Nosrati R, Graham PJ, Zhang B, Riordon J, Lagunov A, Hannam TG, et al. Microfluidics for sperm analysis and selection. Nat Rev Urol. 2017;14:707-30. https://doi.org/10.1038/nrurol.2017.175 
https://www.nature.com/articles/nrurol.2017.175\#supplementaryinformation.

18. Suarez SS, Wu M. Microfluidic devices for the study of sperm migration. MHR: Basic Sci Reprod Med. 2017;23(4):227-34. https://doi.org/10.1093/molehr/gaw039.

19. Eravuchira PJ, Mirsky SK, Barnea I, Levi M, Balberg M, Shaked NT. Individual sperm selection by microfluidics integrated with interferometric phase microscopy. Methods. 2018;136:152-9. https://doi.org/10.1016/j.ymeth.2017.09.009.

20. Samuel R, Feng H, Jafek A, Despain D, Jenkins T, Gale BJTA et al. Microfluidic — based sperm sorting \& analysis for treatment of male infertility 2018. 2018:S336-SS47.

21. Quinn MM, Jalalian L, Ribeiro S, Ona K, Demirci U, Cedars MI, et al. Microfluidic sorting selects sperm for clinical use with reduced DNA damage compared to density gradient centrifugation with swim-up in split semen samples. Hum Reprod(Oxford, England). 2018. https://doi.org/10.1093/humrep/dey239.

22. Huang JYJ, Rosenwaks Z. Assisted reproductive techniques. In: Rosenwaks Z, Wassarman PM, editors. Human fertility: methods and protocols. New York, NY: Springer New York; 2014. p. 171231.

23. Pereira N, Neri QV, Lekovich JP, Palermo GD, Rosenwaks Z. The role of in-vivo and in-vitro maturation time on ooplasmic dysmaturity. Reprod BioMed Online. 2016;32(4):401-6. https:// doi.org/10.1016/j.rbmo.2016.01.007.

24. Neri QV, Lee B, Rosenwaks Z, Machaca K, Palermo GD. Understanding fertilization through intracytoplasmic sperm injection (ICSI). Cell Calcium. 2014;55(1):24-37. https://doi.org/10. 1016/j.ceca.2013.10.006.

25. Irani M, O'Neill C, Palermo GD, Xu K, Zhang C, Qin X, et al. Blastocyst development rate influences implantation and live birth rates of similarly graded euploid blastocysts. Fertil Steril. 2018;110(1):95-102.e1. https://doi.org/10.1016/j.fertnstert.2018. 03.032 .

26. Kuwayama M. Highly efficient vitrification for cryopreservation of human oocytes and embryos: the Cryotop method. Theriogenology. 2007;67(1):73-80. https://doi.org/10.1016/j.theriogenology.2006. 09.014 .

27. Kang H-J. Programmed versus natural frozen embryo transfer: which is the best nest? Fertil Steril. 2018;110(4):636-7. https:// doi.org/10.1016/j.fertnstert.2018.06.020.

28. Brandriff B, Pedersen R. Repair of the ultraviolet-irradiated male genome in fertilized mouse eggs. Science. 1981;211(4489):1431-3. https://doi.org/10.1126/science.7466400.

29. Ashwood-Smith MJ, Edwards RG. Genetics and human conception: DNA repair by oocytes. MHR: Basic Sci Reprod Med. 1996;2(1):46-51. https://doi.org/10.1093/molehr/2.1.46.

30. Esteves SC, Roque M, Bradley CK, Garrido N. Reproductive outcomes of testicular versus ejaculated sperm for intracytoplasmic sperm injection among men with high levels of DNA fragmentation in semen: systematic review and meta-analysis. Fertil Steril. 2017;108(3):456-67.e1. https://doi.org/10.1016/j.fertnstert.2017. 06.018 .

31. Schlegel PN, Su LM. Physiological consequences of testicular sperm extraction. Human reproduction (Oxford, England). 1997;12(8):1688-92.

32. Bucar S, Goncalves A, Rocha E, Barros A, Sousa M, Sa R. DNA fragmentation in human sperm after magnetic-activated cell sorting. J Assist Reprod Genet. 2015;32(1):147-54. https://doi.org/10. 1007/s10815-014-0370-5.

33. Cakar Z, Cetinkaya B, Aras D, Koca B, Ozkavukcu S, Kaplanoglu $\dot{I}$, et al. Does combining magnetic-activated cell sorting with density gradient or swim-up improve sperm selection? J Assist Reprod Genet. 2016;33(8):1059-65. https://doi.org/10.1007/s10815-0160742-0.

34. Shirota K, Yotsumoto F, Itoh H, Obama H, Hidaka N, Nakajima K, et al. Separation efficiency of a microfluidic sperm sorter to minimize sperm DNA damage. Fertil Steril. 2016;105(2):315-21.e1. https://doi.org/10.1016/j.fertnstert.2015.10.023.

35. Amiri I, Ghorbani M, Heshmati S. Comparison of the DNA fragmentation and the sperm parameters after processing by the density gradient and the swim up methods. J Clin Diagnostic Res : JCDR. 2012;6(9):1451-3. https://doi.org/10.7860/JCDR/2012/4198.2530.

36. Aitken RJ, Clarkson JS. Significance of reactive oxygen species and antioxidants in defining the efficacy of sperm preparation techniques. J Androl. 1988;9(6):367-76.

37. Iwasaki A, Gagnon C. Formation of reactive oxygen species in spermatozoa of infertile patients. Fertil Steril. 1992;57(2):409-16.

38. Zini A, Finelli A, Phang D, Jarvi K. Influence of semen processing technique on human sperm DNA integrity. Urology. 2000;56(6): 1081-4.

39. Gil M, Sar-Shalom V, Melendez Sivira Y, Carreras R, Checa MA. Sperm selection using magnetic activated cell sorting (MACS) in assisted reproduction: a systematic review and meta-analysis. J Assist Reprod Genet. 2013;30(4):479-85. https://doi.org/10.1007/ s10815-013-9962-8.

40. Enciso M, Iglesias M, Galán I, Sarasa J, Gosálvez A, Gosálvez J. The ability of sperm selection techniques to remove single- or double-strand DNA damage. Asian J Androl. 2011;13(5):764-8. https://doi.org/10.1038/aja.2011.46.

41. García-Peiró A, Navarro J, Ribas-Maynou J, Benet J, Abad C, Amengual MJ. Alkaline and neutral Comet assay profiles of sperm DNA damage in clinical groups. Hum Reprod. 2012;27(3):652-8. https://doi.org/10.1093/humrep/der461.

42. Ribas-Maynou J, García-Peiró A, Fernandez-Encinas A, Amengual MJ, Prada E, Cortés P, et al. Double stranded sperm DNA breaks, measured by comet assay, are associated with unexplained recurrent miscarriage in couples without a female factor. PLoS One. 2012;7(9):e44679. https://doi.org/10.1371/journal.pone.0044679.

Publisher's note Springer Nature remains neutral with regard to jurisdictional claims in published maps and institutional affiliations. 